\title{
ANÁLISE DA DINÂMICA, ESTRUTURA DE FOCOS EARRANJO ESPACIAL DA MANCHA MANTEIGOSA EM CAMPO ${ }^{1}$
}

\author{
Analysis of the dynamics, foci structure and spatial arrangement of the blister spot in the field
}

\author{
Josimar Batista Ferreira² ${ }^{2}$ Mario Sobral de Abreu ${ }^{3}$, Igor Souza Pereira ${ }^{4}$
}

\begin{abstract}
RESUMO
Objetivou-se estudar a dispersão espacial da mancha manteigosa em cafeeiro, por meio de arranjos espaciais e da análise da dinâmica e estruturas de focos da doença durante três anos consecutivos. Nesse período, não houve progresso da doença no campo. Sob plantas doentes, surgiam mudas com sintomas da doença, um indício da transmissibilidade via semente. Constatou-se um total de 10 focos, com média de 2,5 plantas/focos e com tendência desses focos (maior número de plantas sintomáticas) na direção das linhas de plantios, tendo disposição de forma elíptica. Observou-se número elevado de focos unitários, correspondendo a $52 \%$ das plantas doentes. Pelas análises de sequiências ordinárias runs e doublet verificou-se padrão espacial aleatório. Tal fato indica que a mancha manteigosa ocorre a partir de plantas isoladas (focos unitários) e que a principal via de transmissão é a semente (semente/planta/ semente).
\end{abstract}

Termos para indexação: Colletotrichum gloeosporioides, cafeeiro, epidemiologia, Coffea.

\begin{abstract}
The aim of this study was to analyze the spatial dispersion pattern of the blister spot on coffee trees, through spatial arrangements and the analysis of the dynamics and foci structure of the disease during three consecutive years. No disease progress was observed in this area during the period of study. Seedlings showing disease symptoms were found under the canopy of diseased trees, strongly indicating that the disease may be transmitted through the seeds. Ten foci with 2.5 trees per focus were observed in the studied area. The foci were distributed following the plant rows with elliptical shape. Unitary foci predominated in the area in $52 \%$ of the diseased trees. Analyses of runs and doublets showed a random spatial pattern of the disease. These results evidenced that the blister spot occurs from isolated plants (unitary foci) and this characteristic may possibly be explained if the seeds are the main disease transmission agent (seed-plant-seed).
\end{abstract}

Index terms: Colletotrichum gloeosporioides, coffee trees, epidemiology, Coffea.

(Recebido em 21 de julho de 2006 e aprovado em 2 de junho de 2008)

\section{INTRODUÇÃO}

São poucos os estudos epidemiológicos no patossistema cafeeiro-Colletotrichum e, menos ainda, focando-se a mancha manteigosa. Os sintomas característicos da doença são observados em folhas, inicialmente, manchas de cor verde-clara, de aspecto oleoso e em estágios avançados, as manchas apresentam coloração verde-pálida a amarela e bordas irregulares. Nos ramos e frutos, as lesões são menores, deprimidas, necróticas de cor marrom-claro e bordas irregulares.

Nos últimos anos, a mancha manteigosa tem revelado um agravante na sintomatologia, no campo, observa-se um forte definhamento com grande número de ramos mortos, provocando declínio vegetativo e produtivo (FERREIRA et al., 2005). Desde sua aparição, na Costa Rica, como medida de controle, recomendava-se erradicar todas as plantas doentes, apesar disso, novos casos da doença surgiram (VARGAS \& GONZÁLES, 1972). Atualmente, existem relatos da doença nos estados de Minas Gerais, São Paulo, Paraná, Espírito Santo, Rondônia e Amazonas, nas espécies Coffea arabica L. e Coffea canephora Pierre ex Froenher, nas cultivares Catucaí Vermelho e Amarelo, Rubi, Mundo Novo, Catuaí Vermelho e Conilon (FERREIRA, 2004).

\footnotetext{
${ }^{1}$ Parte da tese apresentada à UNIVERSIDADE FEDERAL DE LAVRAS/UFLA, Caixa Postal 37 - 37200-000, Lavras, MG, pelo primeiro autor, para obtenção do grau de doutor em Agronomia, área de Fitopatologia

Engenheiro Agrônomo, Doutor em Fitopatologia, Professor - Centro Multidisciplinar/CMULT - Universidade Federal do Acre/UFAC - Campus Floresta Estrada Canela Fina, Km 12 - Gleba Formoso, Lote 245 - Colônia São Francisco - 69980-000 - Cruzeiro do Sul, AC - josimarferreira@gmail.com ${ }^{3}$ Engenheiro Agrônomo, Doutor em Fitopatologia, Professor - Departamento de Fitopatologia/DFP - Universidade Federal de Lavras/UFLA - Cx. P. 3037 37200-000 - Lavras, MG - msabreu@ufla.br

${ }^{4}$ Engenheiro Agrônomo, Mestre em Fitopatologia - Departamento de Fitopatologia/DFP - Universidade Federal de Lavras/UFLA - Cx. P. 3037 - $37200-000$ Lavras, MG - igoreloi@yahoo.com.br
}

Ciênc. agrotec., Lavras, v. 33, n. 1, p. 24-30, jan./fev., 2009 
Historicamente, a literatura científica nacional diverge quanto aos danos ocasionados por esse patógeno. O gênero Colletotrichum associado ao cafeeiro é relatado como patógeno primário, que ataca diretamente os tecidos da planta provocando danos e perdas de produções, ora como patógeno secundário. É certo que a ocorrência de Colletotrichum spp. é grave nas regiões cafeeiras do Brasil, pois têm-se observado perdas de produção pela ocorrência da doença em frutos verdes, gerando mumificações com conseqüente queda dos mesmo (FERREIRA et al., 2004). Objetivou-se, neste trabalho, realizar estudos epidemiológicos de dispersão espacial da doença por meio de arranjos espaciais e da análise da dinâmica e estruturas de focos da doença. Essas análises possibilitarão conhecer e entender melhor tal patossistema, gerar hipóteses sobre os processos ecológicos como reprodução, dispersão, sobrevivência, etc., fornecer informação quantitativa sobre a dinâmica populacional do patógeno, além de ser útil para as tomadas de decisão para o manejo do patossistema considerado (BERGAMIN FILHO et al., 2004).

\section{MATERIALE MÉTODOS}

Como área experimental foi utilizada uma lavoura da cv. Catucaí Vermelho, localizada no campus da UFLA, com aproximadamente 0,3ha, com espaçamento de 3,5 x $0,8 \mathrm{~m}$. Nessa área, há uma grande população de plantas que expressam sintomas da mancha manteigosa.

Estudo espacial da doença - Foi realizado o monitoramento da mancha manteigosa, no campo, no período de maio de 2004 a maio de 2006. Foram realizadas avaliações a cada seis meses, verificando-se o número de plantas doentes. Com auxílio do sistema de posicionamento global (GPS), foi mapeada a área experimental, georreferenciando a localização das plantas doentes dentro das linhas de plantios. Os padrões espaciais da doença foram estudados nas linhas de plantio por meio de teste de doublet e teste de run (BERGAMIN FILHO et al., 2004).

Arranjo espacial da doença: análise de doublet Foram caracterizados, nas linhas de plantio os números de plantas sadias (-) e doentes (+). Foi calculado o número esperado de doublets $\mathrm{E}(D)=\mathrm{m}(\mathrm{m}-1) / \mathrm{N}$ e sua variância $\mathrm{s}^{2}(D)=[\mathrm{m}(\mathrm{m}-1)[\mathrm{N}(\mathrm{N}-1)+(2 \mathrm{~N}(\mathrm{~m}-2)+\mathrm{N}(\mathrm{m}-2)(\mathrm{m}-3)-(\mathrm{N}-1) \mathrm{m}(\mathrm{m}-$ $\left.1)] / \mathrm{N}^{2}(\mathrm{~N}-1)\right]$. Calculou-se o valor estandardizado de $\mathrm{Z}_{D}=[(D+0,5-\mathrm{E}(D)] / \mathrm{s}(D)$, com base na distribuição normal, considera-se $Z_{D}>1,64(\mathrm{P}=0,05)$, define-se com padrão agregado e, quando $\mathrm{Z}_{D}<1,64(\mathrm{P}=0,05)$ padrão acaso. Em que: $D$ : número de doublet; m: número de plantas doentes; $\mathrm{N}$ : número de plantas na linha; $\mathrm{s}(D)$ desvio-padrão.

Análise de run - Foi calculado o número esperado de runs $\mathrm{E}(R)=1+2 \mathrm{~m}(\mathrm{~N}-\mathrm{m}) / \mathrm{N}$ e a sua variância $\mathrm{s}^{2}(R)=2 \mathrm{~m}(\mathrm{~N}-$ m) $[2 \mathrm{~m}(\mathrm{~N}-\mathrm{m})-\mathrm{N}] /\left[\mathrm{N}^{2}(\mathrm{~N}-1)\right]$. Calculou-se o valor estandardizado de $\mathrm{Z}_{R}=[R+0,5-\mathrm{E}(R)] / \mathrm{s}(R)$ com base na distribuição normal, considera-se $\mathrm{Z}_{R}>1,64(\mathrm{P}=0,05) \mathrm{com}$ padrão acaso e, quando $\mathrm{Z}_{R}<1,64(\mathrm{P}=0,05)$ padrão agregado. Em que: $R$ : número de runs; m: número de plantas doentes; $\mathrm{N}$ : número de plantas na linha; $\mathrm{s}(R)$ desvio-padrão.

Índice de dispersão - Com base no mapa da distribuição espacial da doença, foram agrupados conjuntos de cinco plantas, contando-se o número de plantas doentes. Em seguida fizeram-se cálculos da variância e da média para designar o índice de dispersão (D), que é a relação entre (Variância ( $\left.\mathrm{s}^{2}\right)$ /Média (x)). Quando $(\mathrm{D}<1)$, indica padrão espacial regular, $(\mathrm{D}=1)$, aleatório e (D>1), agregado.

Análise da dinâmica e estrutura de focos (ADEF) Com base no mapa da distribuição espacial da doença, foram realizados cálculos da análise de estrutura de focos (ADEF) (BERGAMIN FILHO et al., 2004). Consideraramse como foco aquelas plantas com sintomas imediatamente adjacentes no padrão de proximidade vertical, horizontal ou diagonal. Foram avaliados os números de focos unitários (NFU), compreendendo aquele composto por uma planta afetada; estimou-se o número de focos (NF) na área; avaliou-se o número de plantas por focos (NPF); o índice de forma de focos (IFF); o índice de compactação de focos (ICF). Para cada foco designado, quantificou-se o número máximo de linhas (lf) e colunas (lc) ocupadas, sendo utilizados nos cálculos do IFF=[(lf/lc) $]$ e o ICF=[(lf*lc)/ $\mathrm{NPF}$.

\section{RESULTADOS E DISCUSSÃO}

Monitoramento da doença - $\mathrm{Na}$ primeira avaliação, no ano de 2004, foram encontradas 52 plantas doentes, dentre as 423 examinadas. Nos anos de 2005 e 2006, realizaram-se novas avaliações, porém verificou-se padrão similar ao do ano de 2004, não tendo sido constatada a disseminação da doença em campo. Nesse período de monitoramento, observou-se que sob plantas doentes, haviam mudas com sintomas da doença, um forte indício da transmissibilidade via semente. Orozco Miranda et al. (2002a,b) ao estudarem a colonização de Colletotrichum spp. nos tecidos da sementes de plantas doentes com mancha manteigosa, quantificaram, em média, $14 \%$ de infecção por Colletotrichum spp. no endosperma e Ferreira et al. (2004), em estudos de colonização nos ovários, observaram em média, 27,91\% de infecção por Colletotrichum spp.em ovários de plantas com sintomas da mancha manteigosa.

Nesta área de estudo, observa-se a possibilidade de, na formação da lavoura (material em seleção para 
resistência à ferrugem), terem empregado sementes colhidas em plantas doentes, fato que vem explicar o surgimento e a estagnação da doença nessa área, pois é fato que, sob copas de plantas doentes, surgem plântulas com sintomas.

Estudo espacial da doença - A distribuição espaçotemporal de plantas doentes e sadias permaneceu idêntica nas três avaliações realizadas (Figura 1). Em relação ao agrupamento da doença nas linhas de plantios, houve aglomeração máxima de três plantas, verificada em apenas uma das linhas. O maior número de agrupamentos foi de duas plantas doentes consecutivas, num total de sete grupos, observados em seis das oito linhas de plantios (Figura 1). Observou-se também elevado número de focos unitários, num total de 27 focos distribuídos, aleatoriamente, por todas as linhas de plantio (Figura 1).

Dispersão da doença - O índice de dispersão (D) para a área avaliada, (420 plantas agrupadas de cinco em cinco) foi $=0,90$ (média 0,61, variância 0,55). Segundo Bergamin Filho et al. (2004), quando ( $<<1)$ indica padrão espacial regular; $(D=1)$ aleatório; $(D>1)$ agregado. Porém, neste trabalho, com o (D) igual a 0,90 indica padrão espacial regular. O índice de dispersão (D) não foi satisfatório para a descrição do padrão espacial da doença, tendo em vista que, pelo mapa de distribuição (Figura 1) observa-se padrão aleatório, o qual é o mais representativo para esclarecer a dispersão espacial da doença. Segundo Upton \& Fingleton (1985) índices de dispersão (D) são representativos para dados de contagem, como número de lesões por folhas ou número de insetos por plantas. Similarmente ao observado, Laranjeira et al. (2004) quando utilizaram o (D) para estudos da dinâmica espacial da clorose variegada dos citros (CVC), em três regiões do estado de São Paulo, observaram que não houve tendência clara para a elucidação do padrão espacial da doença na maioria das avaliações, com o uso do (D), segundo os mesmos, não foi observado um padrão que, biologicamente, fizesse sentido.

Número de focos e tamanho dos focos - Foram considerados como foco plantas com sintoma da mancha manteigosa imediatamente adjacente em padrão de proximidade vertical, horizontal ou diagonal. Tais critérios são adotados por diversos pesquisadores, nos mais diferentes patossistemas (JESUS JUNIOR \& BASSANEZI, 2004; LARANJEIRA et al., 1998a,b; NELSON, 1996).

Foram mapeadas 423 plantas, das quais 52 estavam doentes com mancha manteigosa, com incidência de 12,3\%. Foi computado o número de focos unitários por linha de plantio, observando, no máximo, sete focos unitários por linha, com média de 3,4 focos unitários por linha de plantio. (Tabela 1). Observou-se número elevado de focos unitários com total de 27 focos, o que correspondente a $52 \%$ das plantas com sintomas da mancha manteigosa (Tabela 1A). $\mathrm{Na}$ área estudada, verificou-se um total de 10 focos, com média de 2,5 plantas por focos, sendo no máximo, quatro plantas e, no mínimo, duas plantas por focos (Tabela 1B). Jesus Junior \& Bassanezi (2004), em estudos da análise da dinâmica e estrutura de focos da morte súbita dos citros, constataram o número máximo de focos em talhões com incidência de 18\%, estimado em 64 focos/1.000 plantas; à medida que aumentou a incidência, diminuía o número de focos por 1.000 plantas. Quando estudaram o número de focos unitários por 1.000 plantas (NF1M), os mesmos autores, verificaram o máximo de focos unitários em talhões
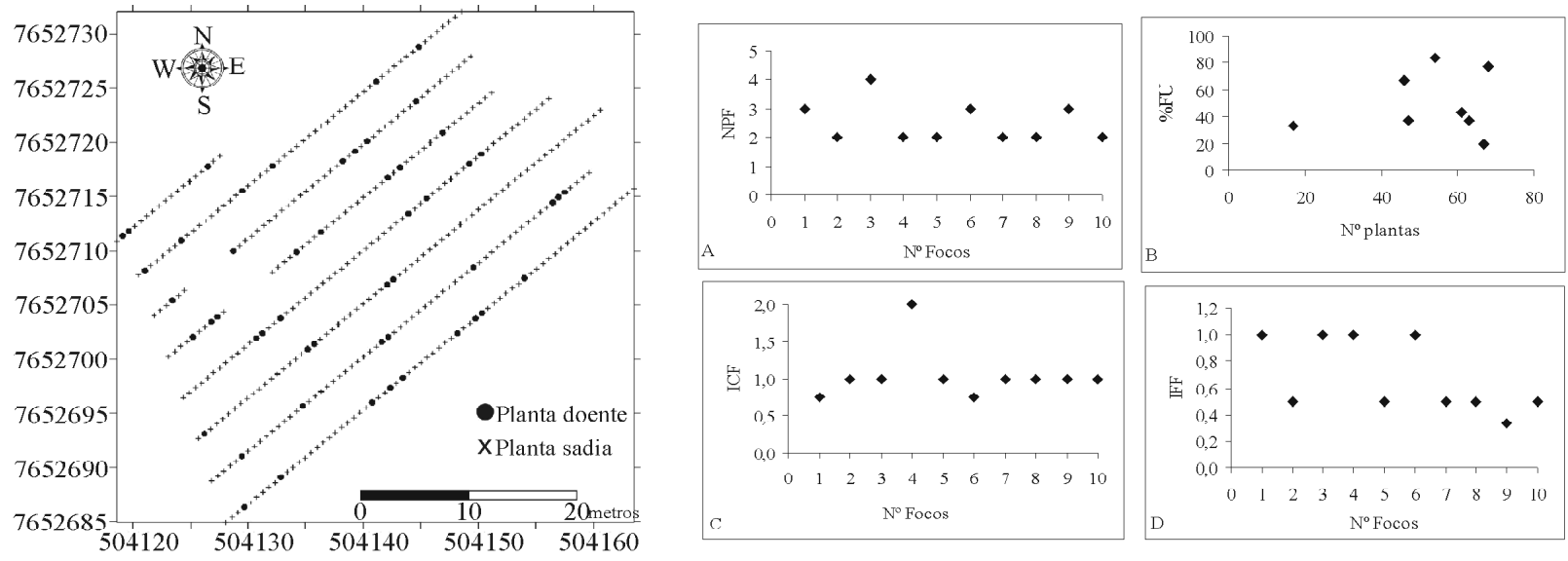

Figura 1 - Mapeamento da distribuição espacial e dinâmica de focos da mancha manteigosa em Coffea arabica L. A: NPF: número de plantas por foco; B: \%FU: porcentagem de focos unitários; C: ICF: índice de compactação de focos; D: IFF: índice de forma de focos. 
com incidência de $13 \%$, estimado em 34 focos/1.000 plantas, diminuindo com o aumento dos níveis de incidência.

No presente trabalho, verificou-se que, na área estudada (incidência de 12,3\%), 52\% eram formados por focos unitários. Tal fato indica que, em geral, o início da epidemia da mancha manteigosa se dá por meio de plantas isoladas, reafirmando que, neste patossistema mancha manteigosa e cafeeiro, a principal via de transmissão da doença é a semente. Verificou-se, que à medida que aumentava o número de plantas observadas, aumentava também o porcentual de focos unitários (Figura 1B). Isso se justifica pelo fato de a transmissão ser, basicamente, via semente, originando plantas adultas doentes em campo, com maior probabilidade de ocorrer focos unitários. Jesus Junior \& Bassanezi (2004), trabalhando com morte súbita do citros, verificaram que, em talhões com até $2 \%$ de plantas com sintomas, $85 \%$ dos focos eram formados por uma única planta e, nos casos de até $10 \%$ de incidência, mais de $65 \%$ dos focos eram unitários. Segundo os autores, o início das epidemias da morte súbita dos citros se dá por meio de plantas isoladas, fato também reportado para clorose variegada dos citros (CVC) (LARANJEIRA et al., 1998a; NELSON, 1996; NUNES et al., 2001), verificando-se que, quando a incidência de CVC foi de $10 \%$, cerca de $60 \%$ dos focos eram unitários.

Como a mancha manteigosa surge em plantas de café? Indaga-se se o fungo está presente nas sementes de plantas enfermas (FERREIRA, 2006; OROZCO MIRANDA et al., 2002a,b), e se tal fonte é a responsável pela disseminação da doença no campo, por meio de mudas formadas a partir sementes colhidas em plantas doentes, as quais originaram plantas adultas a campo. Em vários patossistemas há estudos comprovando a existência e a transmissão de Colletotrichum spp., em semente (MACHADO, 2000; NEERGAARD, 1979; TALAMINI et al., 2002), na cultura do cafeeiro Ferreira (2006) verificou sintomas da mancha manteigosa, em plântulas, a partir de sementes de plantas doentes.

Verificou-se variação quanto ao número de plantas por focos (NPF) (Figura 1A), dos quais 60\% eram formados por duas plantas adjacentes, considerando aproximação vertical, horizontal ou diagonal. Os focos formados por três e quatro plantas equivalem a $30 \%$ e $10 \%$, respectivamente, do total de focos. Diante de tais observações, conclui-se que a mancha manteigosa é uma doença complexa, pois não se tem observado a transmissão planta a planta, tampouco o aumento do número de focos ao longo do tempo (três anos de avaliações). Sabe-se que plantas doentes a campo têm revelado um grande número de ramos mortos, provocando declínio vegetativo e
Tabela 1 - Análise da dinâmica e estrutura de focos (ADEF), avaliados em 0,3 hectares de cafeeiro cv. Catucaí Vermelho infectados com mancha manteigosa. A: número de linhas, número de plantas por linhas, número de plantas doentes (NPD), porcentagem de plantas doentes (\%PD), número de focos unitários (NFU) B: número de focos (NF), número de plantas por focos (NPF), número de plantas doentes na maior linha (lf), número de plantas doentes na maior coluna (lc), índice de forma de focos (IFF) e índice de compactação de focos (ICF).

\begin{tabular}{|c|c|c|c|c|c|}
\hline \multicolumn{6}{|c|}{ A } \\
\hline \multicolumn{2}{|c|}{$\mathrm{N}^{\circ}$ linha } & $\mathrm{N}^{\mathrm{o}}$ plantas & NPD & $\% \mathrm{PD}$ & $\mathrm{NFU}$ \\
\hline \multicolumn{2}{|l|}{1} & 17 & 3 & 17,64 & 1 \\
\hline \multicolumn{2}{|l|}{2} & 54 & 6 & 11,11 & 5 \\
\hline \multicolumn{2}{|l|}{3} & 46 & 6 & 13,04 & 4 \\
\hline \multicolumn{2}{|l|}{4} & 47 & 8 & 17,02 & 3 \\
\hline \multicolumn{2}{|l|}{5} & 61 & 7 & 11,47 & 3 \\
\hline \multicolumn{2}{|l|}{6} & 67 & 5 & 7,46 & 1 \\
\hline \multicolumn{2}{|l|}{7} & 63 & 8 & 12,69 & 3 \\
\hline \multicolumn{2}{|l|}{8} & 68 & 9 & 13,23 & 7 \\
\hline \multicolumn{2}{|c|}{ Área 0,3 ha } & 423 & 52 & 12,3 & $27(52,0 \%)$ \\
\hline \multicolumn{6}{|c|}{$\mathrm{B}$} \\
\hline $\mathrm{NF}$ & NPF & lc & If & IFF & ICF \\
\hline 1 & 3 & 2 & 2 & 1,00 & 0,75 \\
\hline 2 & 2 & 1 & 2 & 0,50 & 1,00 \\
\hline 3 & 4 & 2 & 2 & 1,00 & 1,00 \\
\hline 4 & 2 & 1 & 1 & 1,00 & 2,00 \\
\hline 5 & 2 & 1 & 2 & 0,50 & 1,00 \\
\hline 6 & 3 & 2 & 2 & 1,00 & 0,75 \\
\hline 7 & 2 & 1 & 2 & 0,50 & 1,00 \\
\hline 8 & 2 & 1 & 2 & 0,50 & 1,00 \\
\hline 9 & 3 & 1 & 3 & 0,33 & 1,00 \\
\hline 10 & 2 & 1 & 2 & 0,50 & 1,00 \\
\hline Média & 2,5 & 1,3 & 2 & 0,68 & 1,05 \\
\hline
\end{tabular}

produtivo e que o uso de manejo por meio de recepas não é satisfatório, pois à medida que são emitidas novas brotações, surgem também os sintomas da doença (FERREIRA, 2004; FERREIRA et al., 2005).

Formato e compacidade dos focos - Nesse tipo de análise, segundo Jesus Junior \& Bassanezi (2004), a interpretação da forma e da compacidade dos focos deve ser cuidadosa, principalmente em situações com incidências relativamente elevadas. Porém neste trabalho, a incidência observada foi de $12,3 \%$. Segundo os autores, conforme os índices de incidência, essa começa a interferir 
nos resultados do IFF e do ICF. Por exemplo, normalmente, quando a incidência de plantas doentes é relativamente alta (maior que 35\%), é comum que um foco ocupe todas as linhas ou plantas de uma linha do talhão e que o seu crescimento só ocorra em uma direção por falta de mais linhas ou plantas. Esse tipo de interferência devido à forma do talhão também havia sido reportado por Laranjeira et al. (1998a), em análises de talhões com CVC.

Neste trabalho, pôde-se verificar uma tendência de agrupamento das plantas mais nas linhas do que nas colunas, pelos valores observados entre o número de plantas doentes na maior linha (lf) com o número de plantas doentes na maior coluna (lc) (Tabela 1B). Tal fato ocorreu, provavelmente, devido aos elevados índices de focos compostos por apenas duas plantas adjacentes nas linhas. Para a análise do índice de forma de focos (IFF), foram apenas computados focos com plantas doentes adjacentes, descartando os focos unitários. Verificaramse, na maioria dos focos, valores iguais ou menores que um, em $60 \%$ e $40 \%$ dos casos, sendo na maioria, em torno de 0,5 (média =0,68, desvio-padrão 0,26) (Figura 1D e Tabela 1B). Observou-se que, nos casos em que predominam focos com o número de linha (lf) igual ao número de colunas (lc) ou focos formados por uma única planta, o IFF é igual a 1,0. Segundo Jesus Junior \& Bassanezi (2004), a morte súbita do citros (MSC) mostrou tendências dos valores do IFF serem próximos de a 1,0, principalmente quando se trata de elevado número de focos unitários. Esses mesmo autores analisaram o índice de forma de foco não unitários (IFFNU), o que, segundo eles, permite melhor visualização da forma do foco e uma melhor interpretação do modo de disseminação da doença entre plantas. No presente estudo, também se verificaram altos índices de focos unitários em torno de $52 \%$, que não foram utilizados nos cálculos do IFF.

Mesmo em uma área reduzida, (423 plantas de cafeeiro), foi possível verificar tendência dos focos com maior número de plantas sintomáticas na direção das linhas do que entre linhas de plantios, tendo disposição de forma elíptica e não isodiamétrica, como observado para CVC (LARANJEIRA et al., 1998a; NELSON, 1996). Jesus Junior \& Bassanezi (2004) também verificaram forma elíptica em estudos de dinâmica e estrutura de focos para MSC.

Em referência à compacidade dos focos, verificouse um forte agrupamento, observando-se que a maioria dos focos analisados teve valores próximos de 1,0 (média $=1,05$ e desvio-padrão=0,33), considerados com focos compactos (Figura 1C). Focos mais compactos possuem valores de ICF próximos a 1,0 e os menos compactos, valores menores (NELSON, 1996). Jesus Junior \& Bassanezi (2004), para a MSC, verificaram que à medida que aumentava a incidência, diminuía o ICF.
Comportamento semelhante foi citado por Laranjeira et al. (1998a), para o patossistema CVC. A compacidade observada nas plantas de cafeeiro com sintomas da mancha manteigosa (focos compactos), vem corroborar com as informações de que a via principal da transmissibilidade da doença seja pelas sementes, oriundas de plantas doentes (planta/semente/planta).

Arranjo espacial da mancha manteigosa - Pelas análises de sequiências ordinárias runs, foi constatado em $100 \%$ das linhas de plantio, arranjo espacial aleatório (casual) de plantas doentes nos três anos de monitoramento $(2004,2005$ e 2006) da área (Tabela 2A,B). Verificou-se que o número médio de runs $(R)$ nas linhas de plantios foi 11,6 nas linhas de plantios. Observou-se que o número médio esperado de runs $\mathrm{E}(R)$ foi 11,34 , sendo relativamente próximo ao número de runs observados (Tabela 2A). Com base na distribuição normal, verificouse que os valores estandardizados de $\mathrm{Z}_{R}$ em todas as linhas de plantios foi menor que 1,64, definindo-se como padrão espacial ao acaso, a $5 \%$ de probabilidade.

Com relação às análises de sequiências ordinárias doublet foi constatada a predominância do arranjo espacial aleatório em 62,5\% das linhas de plantios (Tabela 2B). A porcentagem de linhas indicando agregação foi baixa, observada em apenas três das oito linhas de plantio. Tal agregação se deu somente naquelas linhas de plantios em que se observou agrupamento de plantas doentes adjacentes dentro da linha de plantio (Figura 1). Na análise de doublet (D), observou-se número médio de 1,12 doublets nas linhas de plantios, tendo número médio de $\mathrm{E}(D)$ (doublets esperado) igual a 0,71 . Pela análise de doublet, considerando o somatório do número de doublets e o número esperado de doublets $\mathrm{E}(D)$, verificou-se que o valor estandardizado de $\mathrm{Z}_{D}$ foi igual a 1,09 , sendo menor que $1,64(\mathrm{P}=0,05)$ definindo-se como padrão espacial ao acaso (Tabela $2 \mathrm{~B}$ ).

Comparando-se os dois métodos de análises de arranjo espacial (sequiências ordinárias runs e doublet), verificou-se, em ambas as análises, padrão espacial ao acaso (aleatório). Tal fato indica que a mancha manteigosa ocorre por meio de plantas isoladas (focos unitários) e tal característica se deve ao fato de que, nesse patossistema, a principal via de transmissão é semente/planta/semente. Nas observações a campo, Ferreira et al. (2004) verificaram sintomas típicos da doença (mancha clorótica com aspecto oleoso) em plântulas sob plantas enfermas. Eles acreditam que os sintomas da doença se expressam somente em condições especiais de suscetibilidade da planta sendo genética ou não, e/ou da modificação das condições ambientais. Já Vargas \& González (1972) acreditam que, provavelmente, há um caráter genético que predispõe as plântulas a esses sintomas, bem como a uma maior patogenicidade. 
Tabela 2 - Análise de arranjo espacial da mancha manteigosa em cafeeiro Coffea arabica L. analisado por sequiências ordinárias (teste runs e doublet).

\begin{tabular}{|c|c|c|c|c|c|c|c|c|}
\hline \multicolumn{3}{|c|}{$\mathrm{A}$} & \multicolumn{5}{|c|}{ Teste runs } & \multirow{2}{*}{$\begin{array}{c}\mathrm{P}=0,05 \\
\text { Decisão }\end{array}$} \\
\hline linha & $\mathrm{N}$ & $\mathrm{m}$ & $R$ & $\mathrm{E}(R)$ & $\mathrm{s} 2(R)$ & $\mathrm{s}(R)$ & $\mathrm{ZR}$ & \\
\hline 1 & 17 & 3 & 4,0 & 5,0000 & 2,3173 & 1,5222 & $-0,3284$ & casual. \\
\hline 2 & 54 & 6 & 13 & 10,6851 & 4,6070 & 2,1464 & 1,3114 & casual. \\
\hline 3 & 46 & 6 & 13 & 10,4565 & 5,8524 & 2,4191 & 1,2580 & casual. \\
\hline 4 & 47 & 8 & 15 & 13,2978 & 14,6800 & 3,8314 & 0,5747 & casual. \\
\hline 5 & 61 & 7 & 13 & 12,4098 & 6,5519 & 2,5596 & 0,4258 & casual. \\
\hline 6 & 67 & 5 & 7,0 & 9,2686 & 1,6827 & 1,2972 & $-1,3634$ & casual. \\
\hline 7 & 63 & 8 & 11 & 13,9841 & 9,9018 & 3,1467 & $-0,7894$ & casual. \\
\hline 8 & 68 & 9 & 17 & 15,6323 & 13,2513 & 3,6402 & 0,5130 & casual. \\
\hline Total & 423 & 52 & $93\left({ }^{*} 11,6\right)$ & $91,2\left({ }^{*} 11,3\right)$ & 388,7249 & 19,7161 & 0,0904 & casual. \\
\hline \multicolumn{4}{|c|}{$\mathrm{B}$} & \multicolumn{4}{|c|}{ Teste de doublet } & $\mathrm{P}=0.05$ \\
\hline Linha & $\mathrm{N}$ & $\mathrm{m}$ & $D$ & $\mathrm{E}(D)$ & $\mathrm{s} 2(D)$ & $\mathrm{s}(D)$ & $\mathrm{ZD}$ & Decisão \\
\hline 1 & 17 & 3 & 1 & 0,3529 & 0,3395 & 0,5826 & 1,9685 & agreg. \\
\hline 2 & 54 & 6 & 0 & 0,5555 & 0,5522 & 0,7431 & $-0,0747$ & casual. \\
\hline 3 & 46 & 6 & 0 & 0,6521 & 0,6476 & 0,8047 & $-0,1890$ & casual. \\
\hline 4 & 47 & 8 & 1 & 1,1914 & 1,1855 & 1,0888 & 0,2833 & casual. \\
\hline 5 & 61 & 7 & 1 & 0,6885 & 0,6854 & 0,8279 & 0,9801 & casual. \\
\hline 6 & 67 & 5 & 2 & 0,2985 & 0,2967 & 0,5447 & 4,0411 & agreg. \\
\hline 7 & 63 & 8 & 3 & 0,8888 & 0,8855 & 0,9410 & 2,7747 & agreg. \\
\hline 8 & 68 & 9 & 1 & 1,0588 & 1,0555 & 1,0273 & 0,4294 & casual. \\
\hline Total & 423 & 52 & $9\left(^{*} 1,12\right)$ & $6,26\left({ }^{8} 0,71\right)$ & 6,239861 & 2,497971 & 1,093086 & casual. \\
\hline
\end{tabular}

"valores médios; $\mathrm{N}$ : $\mathrm{n}^{\circ}$ plantas; $\mathrm{m}$ : plantas doentes; $\mathrm{R}$ : runs; $\mathrm{E}(R)$ :runs esperados; D: doublet; $\mathrm{E}(D)$ : doublet esperados; $\mathrm{s}^{2}$ : variância; s: desvio-padrão; casual.: casualizado; agreg.: agregado.

Silva et al. (2001), em análise de seqüências ordinárias para descrição do arranjo espacial do vira-cabeça do fumo (Groundnut ringspot virus, GRSV), constataram predominância do arranjo aleatório de plantas doentes e baixo porcentual de agregação nas linhas de plantios. Os arranjos espaciais de plantas com viroses são influenciados pela interação de vários fatores, incluindo ambiente e vetores (MADDEN et al., 1982). Segundo Silva et al. (2001), a predominância do arranjo aleatório de plantas doentes indica que a infecção das plantas de fumo com GRSV pode ter sido originária de uma fonte exógena à área de plantio ou decorrente de mudas infectadas.

\section{CONCLUSÕES}

Não houve disseminação da doença no campo, ao longo do tempo. Verificaram-se poucos focos, com média de 2,5 plantas/focos sendo esses de forma elíptica, com maior número de plantas sintomáticas na direção das linhas do que entre linhas de plantios. Observou-se número elevado de focos unitários correspondendo a 52\% das plantas doentes. Pelas análises de arranjo espacial seqüências ordinárias runs e doublet, verificou-se padrão espacial aleatório da doença a campo. Por meio desses resultados, ditam-se a complexidade dessa doença, pois não se tem observado a transmissão planta a planta, mesmo em condições de plantios adensados com presença de ramos doentes entrelaçados com ramos de plantas sadias, tendo apenas verificado a transmissão por sementes a partir de plantas enfermas.

\section{REFERÊNCIAS BIBLIOGRÁFICAS}

BERGAMIN FILHO, A.; HAU, B.; AMORIM, L.; JESUS JUNIOR, W. C. de. Análise espacial de epidemias. In: VALE, F. X. R. do; JESUS JUNIOR, W. C. de; ZAMBOLIM, L. (Eds.). Epidemiologia aplicada ao manejo de doenças de plantas. Belo Horizonte: Perfil, 2004. p. 195-240. 
FERREIRA, J. B. Flutuação sazonal e associação de Colletotrichum gloeosporioides Penz. a diferentes órgãos e tecidos cafeeiros (Coffea arabica L.). 2004. 90 p. Dissertação (Mestrado em Fitopatologia) - Universidade Federal de Lavras, Lavras, 2004.

FERREIRA, J. B. Aspectos histopatológicos, epidemiologia e controle da mancha manteigosa em Coffea arabica $\mathrm{L}$. 2006. 159 p. Tese (Doutorado em Fitopatologia) Universidade Federal de Lavras, Lavras, 2006.

FERREIRA, J. B.; PEREIRA, I. S.; FERNANDES, K. D.; ABREU, M. S. Prejuízos ocasionados pela mancha manteigosa em cafeeiros (Coffea arabica L.). In: ENCONTRO SUL MINEIRO DE CAFEICULTURA, 10; SIMPÓSIO DE PESQUISA CAFEEIRA DO SUL DE MINAS, 5., 2004, Lavras. Anais... Lavras: Necaf, 2004. CD-ROM.

FERREIRA, J. B.; SILVA, E. H.; FERNANDES, K. D.; PEREIRA, R. B.; ABREU, M. S.; PEREIRA, I. S. Efeito de fungicidas no controle da seca de ramos do cafeeiro $(C$. arabica L.) com mancha manteigosa (Colletotrichum spp.). Fitopatologia Brasileira, Brasília, v. 30, p. 111, 2005. Resumo. Suplemento.

JESUS JUNIOR, W. C. de; BASSANEZI, R. B. Análise da dinâmica e estrutura de focos da morte súbita dos citros. Fitopatologia Brasileira, Brasília, v. 29, n. 4, p. 399-405, jul./ago. 2004.

LARANJEIRA, F. F.; AMORIM, L.; BERGAMIM FILHO, A.; BERGR, R. D.; HAU, B. Análise espacial do amarelinho fatal do dendezeiro como ferramenta para elucidar sua etiologia. Fitopatologia Brasileira, Brasília, v. 23, n. 4, p. 397-403, out./dez. 1998a.

LARANJEIRA, F. F.; BERGAMIM FILHO, A.; AMORIM, L. Dinâmica e estrutura de focos da clorose variegada dos citros (CVC). Fitopatologia Brasileira, Brasília, v. 23, n. 1, p. 36-41, jan./mar. 1998 b.

LARANJEIRA, F. F.; BERGAMIM FILHO, A.; AMORIM, L.; GOTTWALD, T. R. Dinâmica espacial da clorose variegada dos citros em três regiões do estado de São Paulo. Fitopatologia Brasileira, Brasília, v. 29, n. 1, p. 5665, jan./fev. 2004.

MACHADO, J. C. Tratamento de sementes no controle de doenças. Lavras: LAPS/UFLA/FAEPE, 2000. 138 p.
MADDEN, L. V.; LOUIE, R.; ABT, J. J.; KNOKE, J. K. Evaluation of tests of randomness of inflected plants. Phytopathology, Saint Paul, v. 72, n. 2, p. 195-198, Feb. 1982.

NEERGAARD, P. Seed pathology. London: MacMillan, 1979. 839 p.

NELSON, S. C. A simple analysis of disease foci. Phytopathology, Saint Paul, v. 86, n. 4, p. 332-339, Apr. 1996.

NUNES, W. M. C. de; MACHADO, M. A.; CORAZZANUNES, M. J.; FURTADO, E. L. Dinâmica espacial de foco da clorose variegada dos citros (CVC) avaliada por meio da sintomatologia e serologia. Acta Scientiarum, Maringa, v. 23, n. 5, p. 1215-1219, Oct. 2001.

OROZCO MIRANDA, E. F.; FREITAS, M.; PIGOZZO, P.; ABREU, M. S. Incidência de Colletotrichum spp. em frutos cereja e sementes de café arabica (Coffea arabica). In: SIMPÓSIO BRASILEIRO DE PATOLOGIA DE SEMENTES, 7., 2002, Sete Lagoas, MG. Anais... Sete Lagoas, 2002a. p. 59.

OROZCO MIRANDA, E. F.; FREITAS, M.; PIGOZZO, P.; ABREU, M. S. Transmissão de Colletotrichum spp. por sementes de café arabica (Coffea arabica). In: SIMPÓSIO BRASILEIRO DE PATOLOGIA DE SEMENTES, 7., 2002, Sete Lagoas, MG. Anais... Sete Lagoas, 2002b. p. 93.

SILVA, J. N.; MICHEREFF, S. J.; PIO-RIBEIRO, G.; ANDRADE, G. P. Arranjo espacial do vira-cabeça do fumo em Arapiraca, Estado de Alagoas. Fitopatologia Brasileira, Brasília, v. 26, n. 4, p. 721-725, out./dez. 2001.

TALAMINI, V.; POZZA, E. A.; MACHADO, J. C.; OLIVEIRA, F. A. Epidemiologia de doenças associadas a Colletotrichum spp. transmitidas por sementes. Revisão Anual de Patologia de Plantas, Passo Fundo, v. 10, p. 219248, 2002.

UPTON, G.; FINGLETON, B. Spatial data analysis by example: pointe pattern and quantitative data. Chichester: Wiley, 1985. v. 1, 410 p.

VARGAS, G. E.; GONZALEZ, U. L. C. La mancha mantecosa del café causada por Colletotrichum spp. Turrialba, San José, v. 22, n. 2, p. 129-135, Apr./Jun. 1972. 